\title{
CHAPAS LAMINADAS ESPECIFICAÇÕES ASTM-A36, ASTM- A131 GR. A, ASTM- A283 GR. C - PROPRIEDADES QUÍMICAS E MECÂNICAS VALORES COMPARATIVOS
}

\section{LANINATED PLATES SPECIFICATIONS ASTM-A36, ASTM-A131 GR. A, ASTM- A283 GR. C-CHEMICAL AND MECHANICAL PROPERTIES COMPARATIVE VALUES}

\author{
José Francisco Lima \\ Rogério Pereira da Silva II \\ Péricles Bosquetti ${ }^{\text {III }}$ \\ Solange Pereira dos Santos Farah ${ }^{\text {IV }}$ \\ Otávio Contart Gamboni ${ }^{\mathrm{V}}$
}

\begin{abstract}
RESUMO
O fornecimento de chapas laminadas de aço estrutural no Brasil, no período de 1980 a 2005 era na totalidade feito por distribuidores brasileiros de produtos siderúrgicos, os quais são representantes de duas das principais usinas siderúrgicas nacionais: COSIPA e Usiminas. Neste período, cada produto era fabricado atendendo à uma única especificação. Atualmente, com a competitividade, a globalização de mercado, o aumento de produtividade, e a redução de custos e de preço ao consumidor e de excelência em qualidade, evoluiu-se na efetividade de fabricação desses aços de modo que se produza chapas laminadas que possam atender simultaneamente à duas ou três especificações diferentes, desde que, as especificações convirjam à parâmetros que permitam, através de cuidadosos controles, permitam a fabricação destes materiais. Este novo conceito que antes era notado em produtos de outros países, passou a ser praticado por usinas siderúrgicas nacionais como a Gerdau e a Usiminas. Esta nova condição despertou uma curiosidade técnica em avaliar o comportamento deste tipo de material, submetendo-se os certificados da qualidade à uma comparação apurada em suas análises químicas e às propriedades mecânicas obtidas. Assim, neste trabalho, foram avaliados quatro certificados da qualidade de fabricação destes materiais, considerando os valores prescritos pelas respectivas especificações neles contidas, sendo elas, ASTM-A36, ASTM- A131 Gr. A e ASTM- A283 Gr. C. Verificou-se que existe uma equivalência próxima entre os valores especificados, considerando que as faixas especificadas dos principais elementos presentes nestes aços, o carbono e o manganês, elementos estes que refletem diretamente nas propriedades mecânicas, são bem amplas, permitindo-se então, em uma única carga de fabricação (carga forno), conseguir preparar um único material de forma a atender

\footnotetext{
I Tecnólogo em Mecânica: processos de soldagem - Fatec Deputado Waldyr Alceu Trigo de Sertãozinho - SP Brasil. E-mail: jose.lima67@fatec.sp.gov.br

II Tecnólogo em Mecânica: processos de soldagem - Fatec Deputado Waldyr Alceu Trigo de Sertãozinho - SP Brasil. E-mail: rogerio.silva93@fatec.sp.gov.br

III Prof. Dr. na Fatec Deputado Waldyr Alceu Trigo de Sertãozinho - SP - Brasil. E-mail: pericles.bosquetti@fatec.sp.gov.br

IV Profa. Mestre - Fatec Deputado Waldyr Alceu Trigo de Sertãozinho - SP - Brasil. E-mail: solange.farah@fatec.sp.gov.br

v Prof. Dr. - Fatec Deputado Waldyr Alceu Trigo de Sertãozinho - SP - Brasil. E-mail: otavio.gamboni@fatec.sp.gov.br
} 
satisfatoriamente as três especificações, ASTM- A36, ASTM- A131 GR. A e ASTM- A283GR. C.

Palavras-chave: Aços Estruturais. ASTM- A131 Gr. A. ASTM- A283 Gr. C.

\begin{abstract}
The supply of laminated structural steel plates in Brazil, in the period from 1980 to 2005, was entirely made by Brazilian steel products distributors, who are representatives of two of the main national steel mills that until then dominated this market, namely COSIPA and Usiminas. During this period, each product was manufactured according to a single specification separately. Nowadays, with the competitiveness and the new global trends of globalization of the market, of increase of productivity, of reduction of costs and price to the consumer and of excellence in quality, the effectiveness of manufacturing these steels has evolved so that laminated sheets are produced that can simultaneously meet two or three different specifications, provided, of course, that the specifications converge with parameters that allow through careful controls, to allow the manufacture of these materials. This new manufacturing concept, which was previously only noticed in products from other countries, with China highlighted, also started to be practiced by national steel mills, highlighting Gerdau and Usiminas as references. This condition, aroused a technical curiosity in evaluating the behavior of this type of material, subjecting the quality certificates to a refined comparison in their chemical analyzes and the mechanical properties obtained. Thus, in this work, four certificates of the quality of manufacture of these materials were evaluated, considering the values prescribed by the respective specifications contained therein, namely, ASTM-A36, ASTM- A131 Gr. A and ASTM- A283 Gr. C. It was verified that there is a close equivalence between the specified values, considering that the specified ranges of the main elements present in these steels, carbon and manganese, elements that reflect directly on the mechanical properties, are quite wide, allowing then, in a single load manufacturing process (kiln load), to be able to prepare a single material in order to satisfactorily meet the three specifications, ASTM- A36, ASTM- A131 GR. A and ASTM- A283- GR. C.
\end{abstract}

Keywords- Aços Estruturais; ASTM- A131 Gr. A; ASTM- A283 Gr. C.

Data de submissão do artigo: 30/06/2021.

Data de aprovação do artigo: 17/08/2021.

DOI: $10.33635 /$ sitefa.v4i1.180

\title{
1 INTRODUÇÃO
}

Com as novas tendências mundiais de globalização do mercado, a partir dos últimos quinze anos, pôde-se observar no Brasil, e em grande escala, a entrada de produtos fabricados em outros países, flexibilizando-se as opções de compra, o que certamente levou a uma livre concorrência, provocando forte alteração nos preços. Além disso, muitos produtos fabricados em países emergentes em novas e modernas tecnologias como a China, por exemplo, passaram a fazer parte desta comercialização, forçando as empresas siderúrgicas nacionais a buscarem inovações e modernizações em seus processos como opções para maior competitividade, fato que foi sentido de forma notória por profissionais do mercado. 


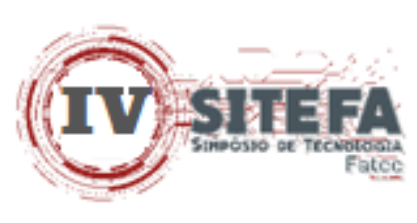

Com esses avanços nos processos, e seguindo essa tendência de melhoria de produtividade e competitividade, principalmente na última década, notou-se que todas as empresas siderúrgicas começaram a implantar produtos no mercado, que atendessem à várias especificações, de forma que um único produto, pudesse a atender três ou até mais diferentes especificações, mesmo sem nenhum documento normativo a respeito. Isto passou a ser tendência em muitos produtos destinados à caldeiraria, sendo chapas e tubos fabricados em aço ao carbono, os mais explorados neste sentido.

Assim, o material adquirido pelo cliente, tendo sua especificação solicitada atendida, além do atendimento às demais especificações, atestadas através de estampa original da siderúrgica no produto e da garantia do respectivo certificado da qualidade, passaram a serem muto bem aceitas pelo mercado. Isto virou tendência na fabricação e fornecimento das usinas siderúrgicas nacionais, até poque também permitem um grande favorecimento logístico dos usuários destes produtos.

\section{FUNDAMENTAÇÃO TEÓRICA}

Visto ser o estudo das principais especificações para aços estruturais o principal foco deste trabalho, faremos um breve levantamento sobre o comitê ASTM, um dos mais importantes organismos elaboradores e regulamentadores de normas técnicas e especificações.

\subsection{Comitê ASTM}

O comitê American Society for Testing and Materials (ASTM) é um órgão sediado nos EUA que tem como responsabilidades: a introdução, o desenvolvimento e a publicação de normas técnicas aplicadas para diversos produtos, materiais, serviços e sistemas organizacionais (ASTM, 2020).

Essa entidade foi criada no ano de 1898 por um grupo de cientistas e engenheiros, e exerce hoje um dos dois maiores papéis entre os padrões de desenvolvedores nos Estados Unidos da América, e é considerada a maior incorporadora mundial de normas, dando suporte a diversos comitês técnicos voluntários (ASTM, 2020). Destaca-se também seu uso como fundamento na criação de normas ou especificações locais de vários países, como é o caso das normas brasileiras ABNT-NBR, em que muitas de suas especificações são baseados em documentos de referência das normas ASTM.

Com milhares de membros espalhados por todo o mundo, são desenvolvidas e mantidas, de forma coletiva, mais de 12.000 normas utilizadas globalmente. Atualmente, conta com 143 comitês principais, e é reconhecida como líder no desenvolvimento e na publicação de normas técnicas e especificações (ASTM, 2020).

Como principais fundamentos e visto a importância para este trabalho que relaciona as especificações para aços estruturais, descrevemos a seguir, a importância das especificações e as peculiaridades de cada uma das três normas para aços estruturais, discutidas neste trabalho, a saber: ASTM- A36, ASTM- A131 Gr. A e ASTM- A283 Gr. C.

\subsection{Especificação}

Documento que estabelece requisitos de necessidade ou expectativa que é expressa, geralmente, de forma implícita ou obrigatória. Uma especificação pode se relacionar a 


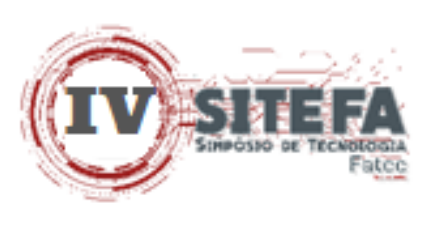

atividades, por exemplo, de ensaios de materiais (ABNT- NBR ISO 9000, 2000). A especificação técnica é um conjunto explícito de exigências a serem satisfeitas por um material, produto ou serviço. Se um material, produto ou serviço não cumprir uma ou mais das especificações aplicáveis, pode ser referido como sendo fora da especificação. Especificações são um tipo de norma técnica. A especificação técnica pode ser desenvolvida por qualquer um dos vários tipos de organizações, tanto públicas como privadas. Tipos de organização são como exemplos: uma corporação, um consórcio (um pequeno grupo de empresas), uma associação industrial (um grupo de toda a indústria das corporações), um governo nacional (incluindo os seus militares, agências reguladoras, e os laboratórios e institutos nacionais), um profissional da associação (sociedade), ou um "fim-made", organização de padrões como a ISO. É comum que uma organização para se referir ou citar os padrões de outra. A especificação de um termo é usada em conexão com uma folha de dados (ou folha de especificações), a qual descreve as características técnicas de um item ou produto. Ela pode ser publicada por um fabricante para ajudar as pessoas a escolher os produtos ou para ajudar a usar os produtos. A ficha de dados não é uma especificação técnica, conforme descrito neste artigo. Em engenharia, fabricação e de negócios, é vital para fornecedores, compradores e usuários de materiais, produtos ou serviços de compreender e concordar com todos os requisitos. A especificação é um tipo de uma norma que é muitas vezes referenciado por um contrato ou documento de aquisição. Ele fornece as informações necessárias sobre os requisitos específicos. As especificações podem serem escritos por agências governamentais, organizações de padrões, associações comerciais, empresas e outros. A especificação do produto não prova necessariamente um produto ser correto para uma dada utilização. Um item pode ser verificado em conformidade com a especificação ou carimbado com um número de especificação: Isto não significa, por si só, indicar que o item é apto para qualquer uso particular, cabendo aos profissionais que usam o item (empresas, instituições, engenheiros, sindicatos, etc.) ou especificar o item (códigos de construção, indústria, governo, etc) têm a responsabilidade de considerar a escolha de especificações disponíveis, especifique o correto, fazer cumprir e use o item corretamente, sendo muitas vezes a validação de adequação se faz necessária. Uma especificação de engenharia adequada, implica necessariamente que todos os produtos fabricados cumpram as metas listadas e tolerâncias. Toda produção real de qualquer material, produto ou serviço envolve variação inerente de saída. A capacidade do processo de materiais e produtos precisa ser compatível com as tolerâncias de engenharia especificado. Os controles do processo devem ser locais, serem adequados e se faz necessário a implementação de um sistema eficaz de gestão da qualidade, tais como a Gestão da Qualidade Total, de forma a garantir e manter a produção real dos materiais dentro das tolerâncias desejadas.

\subsubsection{Especificação ASTM- A36}

É uma especificação criada e desenvolvida pela entidade norte americana ASTM, com as propriedades químicas e mecânicas, citadas na Tabela 1, cujo título é Aço ao Carbono Estrutural (Carbon Structural Steel), e é designada para aplicações diversas. Originariamente foi aprovada e introduzida no mercado no ano de 1960. (CÓDIGO ASTM- SEÇÃO II, 2004). 


\section{(iv)}

Tabela 1 - Composição química e propriedades mecânicas - Especificação ASTM- A36

\begin{tabular}{|c|c|c|c|c|c|c|c|}
\hline \multicolumn{5}{|c|}{ COMPOSIÇÃO QUÍMICA - \% } & \multicolumn{3}{|c|}{ PROPRIEDADES MECÂNICAS } \\
\hline $\begin{array}{c}\text { Carbono } \\
\text { C }\end{array}$ & $\begin{array}{l}\text { Manganês } \\
\text { Mn }\end{array}$ & $\begin{array}{l}\text { Silício } \\
\text { Si }\end{array}$ & $\begin{array}{c}\text { Fósforo } \\
\text { P }\end{array}$ & $\begin{array}{c}\text { Enxofre } \\
\mathrm{S}\end{array}$ & $\begin{array}{c}\text { Limite de } \\
\text { Escoamento }\end{array}$ & & \\
\hline $\begin{array}{l}0,25 \\
\text { máx. }\end{array}$ & - & - & $\begin{array}{l}0,040 \\
\text { máx. }\end{array}$ & $\begin{array}{l}0,050 \\
\text { máx. }\end{array}$ & $\begin{array}{c}250 \mathrm{MPa} \\
\text { mínimo }\end{array}$ & $\begin{array}{c}400 \text { a } 550 \\
\mathrm{MPa}\end{array}$ & $20 \% \mathrm{n}$ \\
\hline
\end{tabular}

Fonte: Adaptado ASTM- A36 (2004)

\subsubsection{Especificação ASTM- A131 Gr. A}

É uma especificação criada e desenvolvida pela entidade norte americana ASTM, com as propriedades químicas e mecânicas, citadas na Tabela 2 cujo título é Aço estrutural para construção naval (Structural Steel for Ships) sendo designada para aplicações dessa natureza. Originariamente foi aprovada e introduzida no mercado no ano de 1931 (CÓDIGO ASTMSEÇÃO II, 2004).

Tabela 2 - Composição química e propriedades mecânicas - Especificação ASTM- A131 Gr. A

\begin{tabular}{|c|c|c|c|c|c|c|c|}
\hline \multicolumn{5}{|c|}{ COMPOSIÇÃO QUÍMICA - \% } & \multicolumn{3}{|c|}{ PROPRIEDADES MECÂNICAS } \\
\hline $\begin{array}{c}\text { Carbono } \\
\text { C }\end{array}$ & $\begin{array}{c}\text { Manganês } \\
\text { Mn }\end{array}$ & $\begin{array}{l}\text { Silício } \\
\text { Si }\end{array}$ & $\begin{array}{c}\text { Fósforo } \\
\text { P }\end{array}$ & $\begin{array}{c}\text { Enxofre } \\
\mathrm{S}\end{array}$ & & & \\
\hline $\begin{array}{l}0,23 \\
\text { máx }\end{array}$ & $\begin{array}{c}2,5 \times \mathrm{C} \\
\text { mín. }\end{array}$ & $\begin{array}{l}0,50 \\
\text { máx. }\end{array}$ & $\begin{array}{l}0,035 \\
\text { máx. }\end{array}$ & & $\begin{array}{c}235 \mathrm{MPa} \\
\text { mínimo }\end{array}$ & $\begin{array}{c}400 \text { a } 520 \\
\mathrm{MPa}\end{array}$ & 21\% mínimo \\
\hline
\end{tabular}

Fonte: Adaptado ASTM- A131 (2004)

\subsubsection{Especificação ASTM- A283 Gr. C}

É uma especificação criada e desenvolvida pela entidade norte americana ASTM, com as propriedades químicas e mecânicas, citadas na Tabela 3, com o título de chapas de aço ao carbono para baixo e médio limite de resistência à tração (Low and intermediate tensile strenght carbon Steel plate), originariamente foi aprovada e introduzida no mercado no ano de 1946 (CÓDIGO ASTM- SEÇÃO II, 2003).

Tabela 3- Composição química e propriedades mecânicas - Especificação ASTM- A283 Gr. C

\begin{tabular}{ccccc|ccc}
\hline \multicolumn{4}{c|}{ COMPOSIÇÃO QUÍMICA - \% } & \multicolumn{3}{c}{ PROPRIEDADES MECÂNICAS } \\
\hline Carbono & Manganês & Silício & Fósforo & Enxofre & Limite de & Limite de & Alongamento \\
C & Mn & Si & P & S & Escoamento & Resistência & \\
\hline 0,24 & 0,90 & 0,40 & 0,035 & 0,040 & $205 \mathrm{MPa}$ & 380 a 515 & $25 \%$ mínimo \\
máx. & máx. & máx. & máx. & máx. & mínimo & $\mathrm{MPa}$ & \\
\hline
\end{tabular}

Fonte: Adaptado ASTM- A283 (2000)

\subsection{Ensaios}

Determinação de uma ou mais características diferenciadoras, como a física, por exemplo, estando envolvidas neste estudo as propriedades químicas e mecânicas (ABNTNBR ISO 9000, 2000). 


\section{(IV) SITEFA}

\subsubsection{Ensaio de análise química}

A análise química avalia as porcentagens de cada elemento químico que compõe o conteúdo do material. É uma dosagem de elementos que intervém nessa composição (COLPAERT, 1994).

\subsubsection{Ensaio de tração}

O ensaio de tração é a deformação de um corpo de prova por uma carga aplicada unixialmente, ao longo do eixo, que aumenta de forma gradativa, até o ponto de fratura, extraindo dele os valores de limite de escoamento, limite de resistência, alongamento e redução de área (CALLISTER, 2018).

\subsubsection{Limite de escoamento}

Quando o material, um metal tensionado, sai de seu limite de regime elástico e entra no processo de deformação plástica, apresentando uma transição gradual, no exato momento, naquele ponto, acontece o limite de escoamento, podendo ser determinado como o ponto onde ocorre o afastamento inicial da linearidade na curva tensão-deformação (CALLISTER, 2018).

\subsubsection{Limite de resistência à tração}

O limite de resistência é a tensão no ponto máximo da curva tensão-deformação de engenharia. Essse ponto corresponde à tensão máxima suportada por um material sob tração. Se essa tensão for aplicada e mantida, ocorrerá fratura (CALLISTER, 2018).

\subsubsection{Alongamento}

O alongamento trata-se da variação do comprimento em um dado instante, tendo como referência o comprimento original. Esta variação não possui unidades, assim o seu valor é expresso em porcentagem. (CALLISTER, 2018). "A ductilidade pode ser expressa quantitativamente tanto como um alongamento percentual quanto como uma redução percentual na área” (CALLISTER, 2018, p. 168).

\subsection{Certificado de Inspeção ou de Qualidade}

Para a avaliação dos lotes dos materiais produzidos, são feitas as inspeções de qualidade, onde ensaios químicos e mecânicos são realizados para avaliação das especificações citadas. Desta forma, emite-se os Certificados de Inspeção ou de Qualidade, que são documento onde são registrados valores de análises, ensaios e outras informações pertinentes, como a especificação do material, controle de fabricação e padrão dimensional. (PETROBRAS N2452, 1996).

\section{PROCEDIMENTOS METODOLÓGICOS}

Utilizou-se como fundamento para a avaliação dos diferentes materiais, a verificação criteriosa dos certificados de inspeção ou de qualidade enviados pelos fabricantes dos aços 


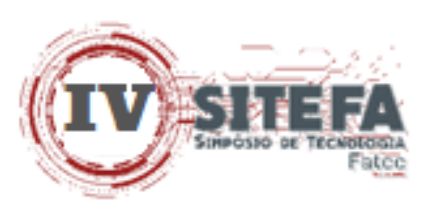

estruturais, com propósitos de se realizar uma avaliação comparativa observando os critérios de análise química e resultados dos ensaios mecânicos como Tensão no Escoamento (LE) e Tensão na Ruptura (LR) bem como a \% Alongamento na ruptura (\%AL). Para obter melhores parâmetros de comparação, foram obedecidos dois critérios fundamentais para melhor caracterizar o trabalho: utilizou-se materiais com a mesma espessura, $3 / 8$ " $(9,5 \mathrm{~mm})$ e sendo procedentes da mesma usina siderúrgica, selecionando-se assim, quatro certificados de inspeção com o propósito de fazer a avaliação comparativa. Os valores obtidos das análises e dos ensaios realizados, foram apresentados nestes documentos junto com os valores previstos pelas respectivas normas, foram disponibilizados na Tabela 5 para facilitar uma analise comparativa. Estes documentos disponibilizados são procedentes da Companhia Siderúgica Usiminas SA. de Ipatinga - MG.

Através de busca de informações junto ao setor de controle integrado de qualidade desta empresa, nos foi esclarecido que este tipo de fornecimento em que um mesmo material tende a outras especificações, como no caso, o atendimento às três especificações para aços estruturais é denominado tríplice certificação, conforme representado na Figura 1, em que produz-se um material dentro de uma faixa mais estreita, mas que cujos valores encontram-se na intersecção das três normas (representado pela região em vermelho da Figura 1). Esta prática passou a ser adotada por opção de mercado, sem uma data inicial, e nem a existência de qualquer documento normativo a respeito deste comportamento, mas que foi possível devido às melhorias de processo de produção, permitindo-se obter melhores e mais precisos controles na composição química e nas características mecânicas destes materiais.

Figura 1- Esquema da tríplice certificação de um material

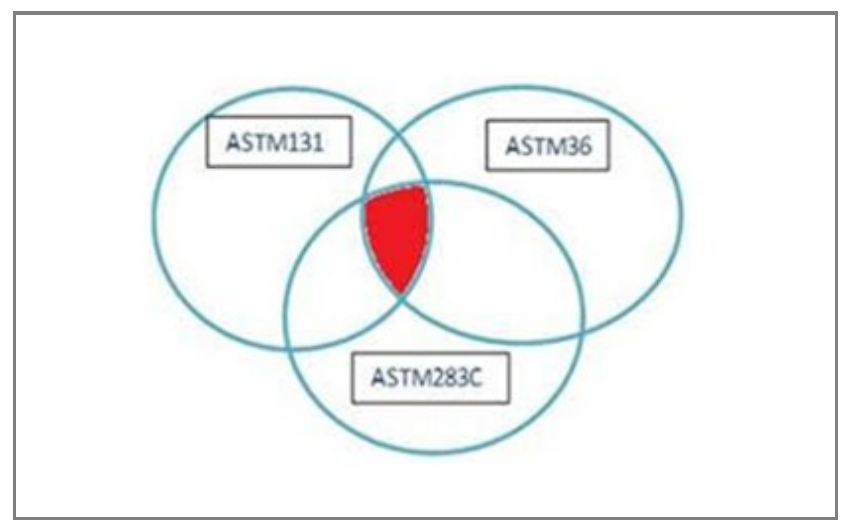

Fonte: Companhia Siderúrgica Usiminas AS (2007)

Como exemplo real desta tríplice certificação, apresentamos na Figura 2 uma chapa de Aço Estrutural em que vê-se letras estampadas numa parte do material nde está descrito as características da mesma. Vê-se que numa das linhas descritas, observa-se a descrição das três especificações ASTM que este material atende.

Figura 2 - Estampa de uma chapa em aço ao carbono

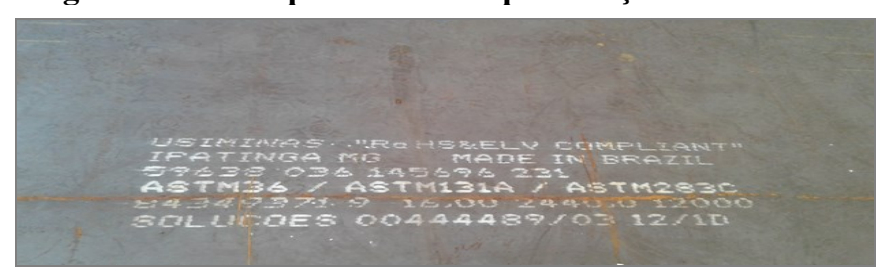

Fonte: Empresa Lince Metalúrgica (2019) 
Para uma melhor caracterização e identificação dos materiais, avaliou-se nos certificados de inspeção avaliados além de valôres técnicos referentes à composição quimica e propriedades mecânicas, também foram extraídas informações essenciais de ordem relevante para esta avaliação, sendo o ano de fabricaçao do material e o número de controle de vazão do forno (corrida). Estas informações estão apresentadas na Tabela 4.

Tabela 4 - Informações complementares

\begin{tabular}{|c|c|c|c|}
\hline ITEM & ESPECIFICAÇÃO & $\begin{array}{c}\text { ANO DE } \\
\text { FABRICAÇÃO }\end{array}$ & No CORRIDA \\
\hline 1 & ASTM- A36 & 2020 & 152040 \\
\hline 2 & ASTM- A131 Gr. A & 2009 & 307566 \\
\hline 3 & ASTM- A283 Gr. C & 2009 & 307566 \\
\hline 4 & A36/ A131 Gr. A/ A283 Gr. C & 2018 & 343977 \\
\hline
\end{tabular}

Fonte: Certificados de inspeção avaliados- Procedência Usiminas ano 2009/2018/2020

A Tabela 5 descrita foi alimentada com as faixas de valores previstos para cada um dos parâmetro citados de composição química e ensaios mecânicoa para cada uma das três especificações ASTM citadas.

Tabela 5 - Composição química e propriedades mecânicas- Especificações ASTM avaliadas

\begin{tabular}{|c|c|c|c|c|c|c|c|c|c|}
\hline \multirow{3}{*}{\multicolumn{2}{|c|}{$\begin{array}{c}\text { FAIXA DE COMPOSIÇÃO } \\
\text { QUÍMICAS E } \\
\text { PROPRIEDADES } \\
\text { MECÂNICAS (ASTM) }\end{array}$}} & \multicolumn{5}{|c|}{ ELEMENTOS QUÍMICOS (\%) } & \multirow{2}{*}{\multicolumn{2}{|c|}{$\begin{array}{c}\text { LE LR } \\
\mathrm{MPa}\end{array}$}} & \multirow{3}{*}{$\begin{array}{c}\text { AL } \\
\text { \% } \\
\text { mín. }\end{array}$} \\
\hline & & \multirow{2}{*}{$\begin{array}{c}\text { C } \\
\text { máx. }\end{array}$} & \multirow{2}{*}{$\begin{array}{c}\text { Mn } \\
-\end{array}$} & \multirow{2}{*}{$\begin{array}{c}\text { Si } \\
\text { máx. }\end{array}$} & \multirow{2}{*}{$\begin{array}{c}\mathbf{P} \\
\text { máx. }\end{array}$} & \multirow{2}{*}{$\begin{array}{c}\text { S } \\
\text { máx. }\end{array}$} & & & \\
\hline & & & & & & & mín. & Faixa & \\
\hline & - Valores previstos pela & & & & & & & & \\
\hline 1 & ASTM- A36 & 0,25 & - & - & & & 250 & $\begin{array}{l}400 \\
550\end{array}$ & 20 \\
\hline 2 & ASTM- A131 Gr. A & 0,23 & $\begin{array}{c}2,5 \mathrm{xC} \\
\text { mín. }\end{array}$ & 0,50 & 0,035 & 0,035 & 235 & $\begin{array}{l}400 \\
520\end{array}$ & 21 \\
\hline 3 & ASTM- A283 Gr. C & 0,24 & $\begin{array}{l}0,90 \\
\text { máx. }\end{array}$ & 0,40 & 0,035 & 0,040 & 205 & $\begin{array}{l}380 \\
515\end{array}$ & 25 \\
\hline
\end{tabular}

Fonte: Valores extraídos das respectivas Normas ASTM (2000; 2004; 2004)

\section{RESULTADOS E DISCUSSÕES}

Os valores dos resultados obtidos nas análises químicas e dos ensaios de tração, extraídos de cada um dos quatro (04) certificados de inspeção avaliados, estão apresentados na Tabela 6. Destes certificados, temos que os três primeiros atendem apenas a uma das especificações ASTM, enquanto que em um deles, está certificado o atendimento à tríplice especificação.

Avaliando-se minuciosamente os valores encontrados em cada certificado de inspeção apresentado, é notória a aproximação entre as três especificações em discussão, pois as faixas solicitadas por cada uma delas, como a porcentagem de carbono e manganês, elementos que refletem diretamente nas propriedades mecânicas, são bem amplas, permitindo à usina fabricante, alinhar uma faixa de composição química, bem como das propriedades mecânicas finais, ajustando ainda na produção da carga de forno a ser vazada, a composição química e 


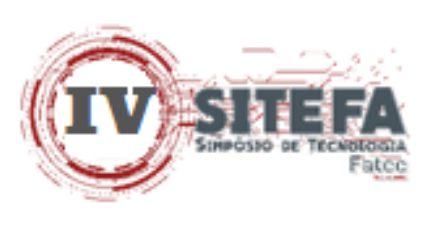

controles adequados de pós-solidificação e pós-tratamentos térmicos de forma a assegurar o atendimento das propriedades mecânicas das especificações.

Tabela 6 - Composição química e propriedades mecânicas-Certificados de Corridas de materiais avaliados

\begin{tabular}{|c|c|c|c|c|c|c|c|c|}
\hline \multirow{3}{*}{$\begin{array}{c}\text { CORRIDAS DOS MATERIAIS } \\
\text { E ATENDIMENTO ÀS } \\
\text { NORMAS (USIMINAS) }\end{array}$} & \multicolumn{5}{|c|}{$\begin{array}{c}\text { ELEMENTOS QUÍMICOS } \\
(\%)\end{array}$} & \multirow{2}{*}{\multicolumn{2}{|c|}{ 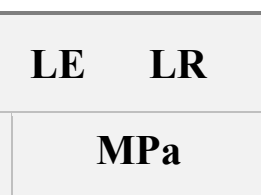 }} & \multirow{2}{*}{$\begin{array}{r}\text { AL } \\
\%\end{array}$} \\
\hline & $\mathbf{C}$ & Mn & $\mathbf{S i}$ & $\mathbf{P}$ & $\mathbf{S}$ & & & \\
\hline & máx. & - & máx. & & máx. & mín. & Faixa & mín. \\
\hline
\end{tabular}

- Valores obtidos nos certificados de inspeção individuais

\begin{tabular}{l|l|lllll|lll}
\hline $\mathbf{1}$ & Corrida 152040 ASTM- A36 & 0,16 & 0,80 & 0,22 & 0,025 & 0,010 & 307 & 467 & 28 \\
$\mathbf{2}$ & Corrida 307566 ASTM-A131 Gr.A & 0,14 & 0,81 & 0,21 & 0,015 & 0,080 & 304 & 456 & 30 \\
$\mathbf{3}$ & Corrida 307566 ASTM-A283 Gr.C & 0,14 & 0,81 & 0,21 & 0,015 & 0,080 & 304 & 456 & 30
\end{tabular}

- Valores obtidos no certificado de inspeção único com as três especificações

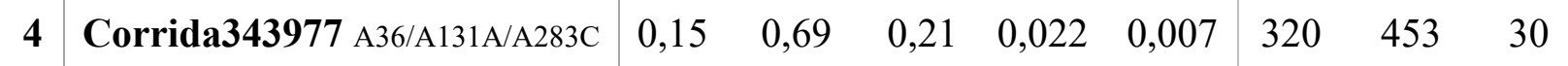

Fonte: Certificados de inspeção avaliados- Procedência Usiminas - anos 2009/2018/2020

Avaliando-se minuciosamente os valores encontrados em cada certificado de inspeção apresentado, é notória a aproximação entre as três especificações em discussão, pois as faixas solicitadas por cada uma delas, como a porcentagem de carbono e manganês, elementos que refletem diretamente nas propriedades mecânicas, são bem amplas, permitindo à usina fabricante, alinhar uma faixa de composição química, bem como das propriedades mecânicas finais, ajustando ainda na produção da carga de forno a ser vazada, a composição química e controles adequados de pós-solidificação e pós-tratamentos térmicos de forma a assegurar o atendimento das propriedades mecânicas das especificações.

Uma observação verificada foi que no certificado do item 1 da Tabela 4, mesmo o material sendo produzido no ano de 2020, apresentou apenas a garantia de uma única especificação, no caso, a ASTM- A36, fato que não tem sido regra, visto que a tendência recente é sempre certificar as três especificações em um mesmo material.

Outro fato que também chamou a atenção, foram os itens 2 e 3 da mesma Tabela 4, onde os materiais foram certificados duas especificações de forma individual, com uma única vazão de forno, ou seja, com a corrida de número 307566, foram comercializadas chapas garantindo especificações separadas, sendo, em ASTM- A131 Gr. A, assim como também em ASTM- A283 Gr. C.

\section{CONSIDERAÇÕES FINAIS}

A intenção dessa verificação foi buscar semelhanças seguras entre as três especificações avaliadas para aços estruturais, para atestar que o procedimento adotado, de disponibilizar os materiais para o mercado atendendo à todas, estaria também satisfazendo as necessidades de cada consumidor quanto à aplicação final do produto. Pôde-se então atestar a proximidade entre as faixas especificadas previstos com os valores reais encontrados. 


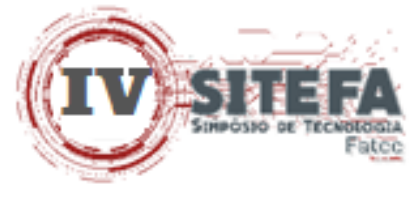

Verificou-se que, tendo as normas amplas faixas para trabalhar com a dosagem dos principais elementos químicos, assim como para as propriedades mecânicas, foi possível a fabricação de um único produto contemplar as especificações ASTM- A36, ASTM- A131 Gr, A e ASTM- A283 Gr. C.

Um fator muito importante a ser considerado, levando-se em conta a utilização destes materiais é a sua condição de soldabilidade. Estas são proporcionadas por cada especificação, e que, conforme prescrito na norma ASME IX, estas três especificações de aços estruturais pertencem ao mesmo grupo de classificação para soldagem, e assim, recebem os mesmos critérios e parâmetros para esse processo de união (ASME IX, 2019). Desta forma, o material produzido atendendo às três especificações, devem atender aos requisitos de processabilidade e qualidade exigidos para esses materiais.

\section{REFERÊNCIAS}

AMERICAN SOCIETY FOR TESTING AND MATERIALS. ASTM- A36. 2004 ed. West Conshohocken: ASTM International., 2004.

ASTM- A131 GR. A. 2004 ed. West Conshohocken: ASTM International., 2004.

ASTM- A283 GR. C. 2003 ed. West Conshohocken: ASTM International., 2004.

AMERICAN SOCIETY OF MECHANICAL ENGINEERS. ASME IX. 2019 ed. New York: ASME Boiler and Pressure Vessel Code An International, 2019.

ASSOCIAÇÃO BRASILEIRA DE NORMAS TÉCNICAS- NORMA BRASILEIRA REGISTRADA- INTERNATIONAL STANDARD ORGANIZATION- ABNT- NBR ISO 9000, 2000 ed. São Paulo: Tecnologia gráfica - Impressos de segurança.,2000.

CALLISTER, W. D. Jr; RETHWISCH, D. G. Ciência e Engenharia dos Materiais. 9. ed. Rio de Janeiro: LTC, 2018. p. 168.

CERTIFICADO DE INSPEÇÃO. Chapa ASTM- A36 espessura 9,50 mm. 2020. ed. Ipatinga: Usiminas, 2020. Número 5247285.

Chapa ASTM- A131 Gr. A espessura 9,50 mm. 2009. ed. Ipatinga: Usiminas, 2009. Número 2138284.

Chapa ASTM- A283 Gr. C espessura 9,50 mm, 2009. ed. Ipatinga: Usiminas, 2009. Número 2130797.

Chapa ASTM- A36, ASTM- A131 Gr. A, ASTM- A283 Gr. C espessura 9,50 mm, 2018. ed. Ipatinga: Usiminas, 2018.

COLPAERT, H. Metalografia dos Produtos siderúrgicos comuns. 2. ed. São Paulo: Edgard Blucher Ltda., 1994.

NORMA PETROBRAS. N-2452. Apresentação de certificado de Ensaio. 1996. ed. Rio de Janeiro: CONTEC Comissão de Normas Técnicas, 1996. 